\title{
Time-resolved high-harmonic spectroscopy of nonadiabatic dynamics in $\mathrm{NO}_{2}$
}

\author{
P. M. Kraus, ${ }^{1}$ Y. Arasaki, ${ }^{2}$ J. B. Bertrand,${ }^{3}$ S. Patchkovskii, ${ }^{3}$ P. B. Corkum, ${ }^{3}$ D. M. Villeneuve, ${ }^{3}$ \\ K. Takatsuka, ${ }^{2}$ and H. J. Wörner ${ }^{1, *}$ \\ ${ }^{1}$ Laboratorium für Physikalische Chemie, ETH Zürich, Wolfgang-Pauli-Strasse 10, 8093 Zürich, Switzerland \\ ${ }^{2}$ Department of Basic Science, Graduate School of Arts and Sciences, The University of Tokyo, Komaba 153-8902, Tokyo, Japan \\ ${ }^{3}$ Joint Attosecond Science Laboratory, National Research Council of Canada and University of Ottawa, \\ 100 Sussex Drive, Ottawa, Canada, KlA0R6
}

(Received 21 September 2011; published 12 April 2012)

\begin{abstract}
Time-resolved high-harmonic spectroscopy is an emerging approach to measuring coupled electronic and nuclear dynamics in photochemical reactions. A general conceptual and theoretical model for the technique is derived from first principles and applied to study the sensitivity of the technique to nonadiabatic dynamics. By comparing the model with detailed experimental data on photoexcited $\mathrm{NO}_{2}$ molecules, we find that time-resolved high-harmonic spectroscopy is primarily sensitive to electronic population dynamics. The coordinate dependence of the vertical ionization potential and photorecombination matrix elements contribute also, but much less significantly, to the observed dynamics because of the rapid spreading of the wave packet in the excited state. We discuss the extension of the method to larger polyatomic molecules in light of this insight.
\end{abstract}

DOI: 10.1103/PhysRevA.85.043409

PACS number(s): 33.20.Xx, 42.65.Ky, 82.53.Eb, 82.53.Kp

In the past, high-harmonic spectroscopy (HHS) has been developed into a powerful approach to studying the structure and dynamics of molecules [1-4]. Since the underlying process is driven by an ultrashort laser pulse, the technique is ideally suited for the investigation of the fastest molecular processes, especially electronic dynamics. Recently, we have reported the application of HHS to a photochemical reaction, the photodissociation of $\mathrm{Br}_{2}$, using either a traditional collinear pump-probe scheme [5] or a transient grating geometry [6]. The latter approach takes advantage of the natural interference between the emission of excited and unexcited molecules and allows an extraction of the relative high-harmonic amplitudes and phases of the species. The studies of $\mathrm{Br}_{2}$ showed that HHS is particularly sensitive to the variation of the vertical ionization potential along the reaction coordinate, through the phase of the emitted harmonics, but also to the electronic structure of the reacting molecule through the photorecombination matrix elements [5-9]. In contrast to $\mathrm{Br}_{2}$, the vast majority of photochemical processes involve more than one electronic state, and HHS is naturally suited to study the associated electronic dynamics on femtosecond to attosecond time scales.

Recently, we have applied time-resolved HHS to a nonadiabatic process, the photodissociation of $\mathrm{NO}_{2}$. Following photoexcitation at $400 \mathrm{~nm}$, we probed through HHS the dynamics of the excited-state wave packet as it crossed the conical intersection [10]. In this article, we derive a quantitative model of time-resolved HHS and use it to study the sensitivity of the technique to electronic and nuclear dynamics. We present additional experimental data and a general theoretical approach relying on a multichannel model of high-harmonic emission and wave-packet calculations in full dimensionality. Our model reproduces all characteristic aspects of the experiment and allows us to conclude that, in $\mathrm{NO}_{2}$, HHS probes femtosecond electronic population dynamics at conical intersections.

\footnotetext{
*woerner@phys.chem.ethz.ch; www.atto.ethz.ch
}

The photochemistry of $\mathrm{NO}_{2}$ has been extensively studied previously ( [11] and references therein), but the femtosecond dynamics following single-photon excitation at $400 \mathrm{~nm}$ has only been observed recently [10]. Cuts of the relevant potential energy surfaces of $\mathrm{NO}_{2}$ and $\mathrm{NO}_{2}^{+}$along the bending and a single NO bond-stretch coordinates are shown in Figs. 1(a) and $1(\mathrm{~b})$, respectively. The $\tilde{X}^{2} A_{1}$ ground and the $\tilde{A}^{2} B_{2}$ electronically excited states of $\mathrm{NO}_{2}$ have the dominant configurations $(\ldots)\left(b_{2}\right)^{2}\left(a_{1}\right)^{1}$ and $(\ldots)\left(b_{2}\right)^{1}\left(a_{1}\right)^{2}$, respectively. The singly occupied molecular orbitals in these two configurations are shown above the potential energy surfaces. We note that their symmetry properties for rotation around the $z$ axis $\left(C_{2}\right.$ symmetry axis) is opposite, which will affect the polarization dependence of the measurements described below. The potential energy surfaces of the $\tilde{X}^{2} A_{1}$ and $\tilde{A}^{2} B_{2}$ states are degenerate along a one-dimensional seam of conical intersections spanning the symmetric stretch coordinate (not shown). Single-photon excitation close to $400 \mathrm{~nm}$ prepares a wave packet in the ${ }^{2} B_{2}$ state, which initially moves along the bending coordinate toward the conical intersection, then crosses the intersection several times before returning into the ${ }^{2} A_{1}$ ground state while spreading along the stretching coordinates as further detailed in the calculations below. The transition dipole moment for the $\tilde{A}^{2} B_{2} \leftarrow \tilde{X}^{2} A_{1}$ transition lies parallel to the $y$ axis (O-O axis, see Fig. 1). Photoexcited molecules thus have their $y$ axis aligned parallel to the polarization of the pump pulse (with a $\cos ^{2}$ distribution). In this article, we experimentally and theoretically study the manifestations of the wave packet crossing the conical intersection, investigating which aspects of the coupled electronic and nuclear dynamics HHS is most sensitive to.

The experimental setup has been described in detail previously [6,10]. Briefly, two synchronized 400-nm pump pulses (40 fs, $10 \mu \mathrm{J}$ ) propagate parallel to an $800-\mathrm{nm}$ probe pulse (32 fs, $0.6 \mathrm{~mJ}$ ) with a vertical offset of $\pm 0.75 \mathrm{~cm}$ onto a $f=50 \mathrm{~cm}$ curved mirror that focuses them into a pulsed $\mathrm{NO}_{2}$ gas jet ( 1 bar backing pressure) heated to $100^{\circ} \mathrm{C}$. The pump pulses set up a transient grating of excitation in the vertical direction, and the probe pulse generates high-order 
polarizations of laser pulses:

pump $(400 \mathrm{~nm}) \quad$ probe $(800 \mathrm{~nm})$
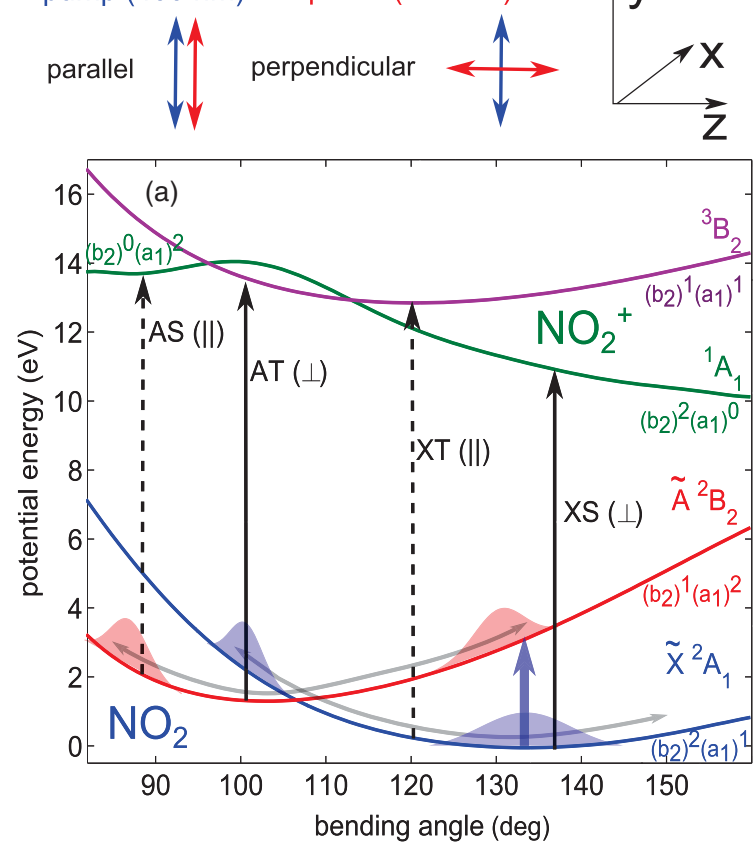

singly-occupied molecular orbitals:

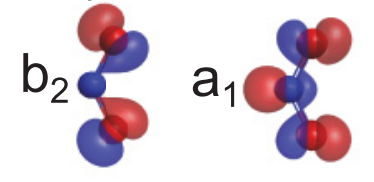

(b)

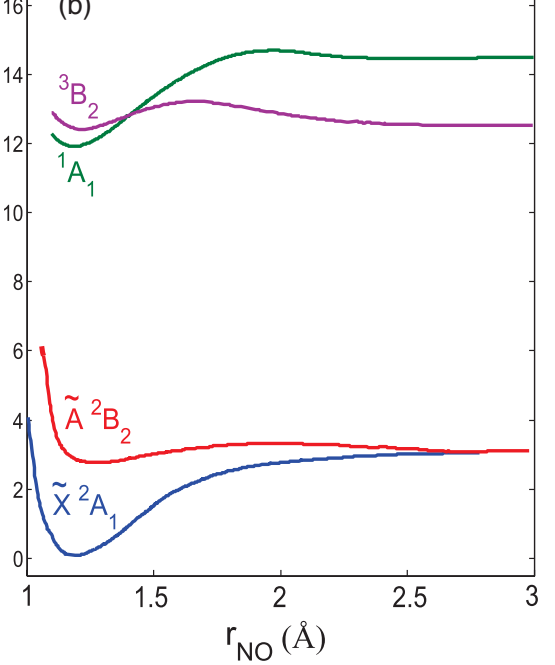

FIG. 1. (Color online) Potential energy surfaces and dominant electronic configurations of the electronic states of $\mathrm{NO}_{2}$ and $\mathrm{NO}_{2}^{+}$relevant for this study as a function of the bending angle ((a), this work) or one N-O bond length ((b), from Ref. [11]). The most weakly bound Hartree-Fock orbital wave functions of $a_{1}$ and $b_{2}$ symmetries are shown as isoamplitude surfaces with a color-coded sign. Photoexcitation at $400 \mathrm{~nm}$ prepares a wave packet in the $\tilde{A}^{2} B_{2}$ state which transfers progressively into the $\tilde{X}^{2} A_{1}$ state, as further illustrated in Fig. 3 . The dominant channels for high-harmonic emission in the case of perpendicular $(\perp)$ or parallel (\|) polarizations of the pump and probe pulses are indicated.

harmonics that are spectrally dispersed in the horizontal plane by a concave grating, whereas the beam freely diverges in the vertical direction. The periodic structure of the medium results in partial first-order diffraction $(m= \pm 1)$ in addition to the nondiffracted component $(m=0)$ of each harmonic order. The spectrally resolved far-field harmonic profiles are detected with a microchannel plate detector backed with a phosphor screen and recorded with a charge-coupled device camera. Measurements have been carried out using either parallel polarizations of pump and probe pulses or perpendicular polarizations, as illustrated at the top of Fig. 1.

The experimental results, taken under conditions similar to Ref. [10], are shown in Fig. 2. Before discussing the observation of molecular dynamics, we explain the generic features observed in two-color transient-grating measurements. The temporal overlap of the pump and probe pulses leads to wave mixing (see Ref. [12]), which causes the appearance of even harmonics as shown in Figs. 2(c) and 2(d) and also contributes to the intensity of the odd harmonics. This contribution is much larger for parallel polarizations of the 400- and 800-nm pulses than in the case of perpendicular polarizations [12]. Hence, wave mixing contributes very weakly to the odd-harmonic signals around time zero in panels 2(a) and 2(c), whereas it dominates these signals in panels 2(b) and 2(d). In the latter case, the contribution to the diffracted component $(m=1)$ is constructive in all cases, whereas the contribution to $m=0$ is constructive (H13) or destructive (H17) depending on the harmonic order. This behavior can be understood from the power scaling of wave mixing [12] and will not be discussed further here.
We now turn to the temporal evolution of the high-harmonic signals outside the region of temporal overlap. In the case of perpendicularly polarized pump and probe pulses [panels 2(a) and 2(c)], the intensity of the diffracted harmonic radiation shows damped oscillations over the first 300 fs, followed by a smooth variation. A similar although much weaker modulation with an opposite phase is observed in the nondiffracted part of the emission $(m=0)$. Very weak modulations are also observed in the case of parallel polarizations of pump and probe pulses [panels 2(b) and 2(d)], both in the nondiffracted and diffracted harmonic yields. In the following, we compare these observations to calculations.

To investigate the origin of the observed oscillations, we first discuss the wave-packet calculations. These calculations were done following the method described in Refs. [13,14] using a 40-fs excitation pulse centered at $400 \mathrm{~nm}$. One-photon excitation prepares a wave packet on the $\tilde{A}^{2} B_{2}$ state (called "state $A$ " in the following) which moves toward the conical intersection. Figure 3(a) shows the population of the diabatic state $A$ as a function of time, together with the expectation value of the bond angle $\left\langle\chi_{i}|\beta| \chi_{i}\right\rangle$, where $i=X, A$ labels the diabatic state and $\chi_{i}$ is the nuclear wave packet associated with the diabatic state $i$. At delays corresponding to maxima and minima of the population in the $A$ state, probability-density distributions of the wave packets in the two diabatic states are shown. At a delay of $11 \mathrm{fs}$, corresponding to the first maximum of the diabatic population, the leading edge of the wave packet has crossed the conical intersection without significantly transferring to state $X$. The wave packet in state 

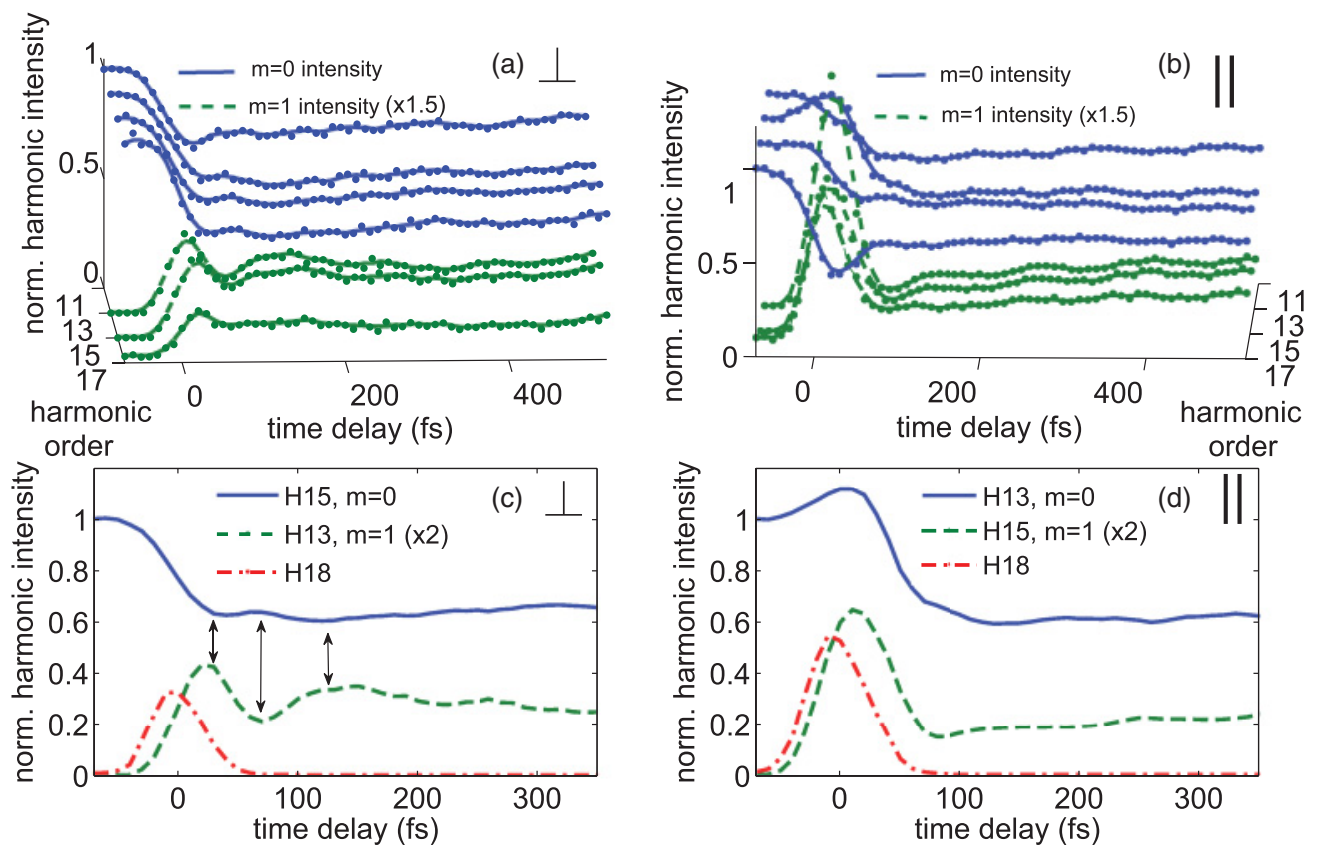

FIG. 2. (Color online) Experimental data from high-harmonic transient grating spectroscopy in $\mathrm{NO}_{2}$. The panels show the measured diffracted $(m=+1)$ and nondiffracted $(m=0)$ high-harmonic intensities as a function of the pump-probe delay, normalized to the $m=0$ signal at negative time delays. The data has been recorded with perpendicular polarizations of pump and probe pulses [panels (a) and (c)] or parallel polarizations [panels (b) and (d)].

$A$ is significantly delocalized along both the $\beta$ and the $r_{1}$ (shown) and $r_{2}$ bond-length coordinates (not shown). The large spread along the bond-stretch coordinates, in particular along the $\mathrm{b}_{2}$ asymmetric stretching mode responsible for vibronic coupling between states $X$ and $A$, leads to a strong transfer of the population from state $A$ to state $X$ when the bulk of the wave packet returns to the conical intersection from the small-bond-angle side. This results in the first minimum in the diabatic population of state $A$, as is clearly visible in the probability-density distribution at 51 fs. The subsequent maxima and minima correspond to oscillations of the wave packet on the lower adiabatic sheet of the potential energy surface, corresponding to strong transfers between the diabatic states.

We now turn to model the observables of time-resolved HHS, generalizing Refs. [5,6] to polyatomic molecules. The pump pulse prepares each $\mathrm{NO}_{2}$ molecule in a superposition state of the form

$$
\Psi(\mathbf{r}, \mathbf{R}, t)=c_{g}(t) \phi_{g}(\mathbf{r} ; \mathbf{R}) \chi_{g}(\mathbf{R})+\sum_{i=X, A} c_{i}(t) \phi_{i}(\mathbf{r} ; \mathbf{R}) \chi_{i}(\mathbf{R}, t),
$$

where $\mathbf{r}$ and $\mathbf{R}$ represent the electronic and nuclear coordinates, respectively, and $\phi$ and $\chi$ the (normalized) electronic and nuclear wave functions at the time delay $t$ elapsed since excitation. Defining the excited-state fraction as $r=1-$ $\left|c_{g}\right|^{2}=\sum_{i}\left|c_{i}\right|^{2}$ and $\left|c_{i}\right|^{2}=r_{i}$, the high-harmonic electric field emitted by this superposition state is given by

$$
E_{\mathrm{XUV}}(\Omega, t)=[1-r(t)] \tilde{d}_{g}(\Omega)+\sum_{i=X, A} r_{i}(t) \tilde{d}_{i}(\Omega, t),
$$

where $\tilde{d}_{i}(\Omega, t)$ represents the time-dependent complex spectral component of high-harmonic emission at photon frequency $\Omega$ for the wave packet in state $i$. The transient grating induces a spatial modulation of the excitation fraction $r_{\bmod }(x)=$ $r[\cos (k x)+1]$ in the direction $(x)$ perpendicular to the propagation of the probe beam $(z)$, where $k=2 \pi / \Lambda$ and $\Lambda=13.3 \mu \mathrm{m}$ is the period of the grating. In the far field, the intensity of the nondiffracted and diffracted components of the emission is thus determined to be

$$
\begin{gathered}
I_{m=0}(\Omega, t)=\left|[1-r(t)] \tilde{d}_{g}(\Omega)+\sum_{i=X, A} r_{i}(t) \tilde{d}_{i}(\Omega, t)\right|^{2}, \\
I_{m= \pm 1}(\Omega, t)=\frac{1}{4}\left|\sum_{i=X, A} r_{i}(t)\left[\tilde{d}_{i}(\Omega, t)-\tilde{d}_{g}(\Omega)\right]\right|^{2} .
\end{gathered}
$$

The high-harmonic emission from each wave packet is calculated in a basis of diabatic electronic states because their electronic character varies slowly with nuclear displacements. The complex spectral component of high-harmonic emission for wave packet $i=g, X, A$ is given by [5,6]

$$
\tilde{d}_{i}(\Omega, t)=\int d \mathbf{R}\left|\chi_{i}(\mathbf{R}, t)\right|^{2} \sum_{f} \sqrt{I^{i f}(\mathbf{R})} e^{-i I_{p}^{i f}(\mathbf{R}) \tau} \tilde{d}_{r}^{i f}(\mathbf{R}),
$$

where the sum runs over the accessible states $f$ of the cation. $I^{i f}$ is the rate of strong-field ionization from state $i$ to the cation state $f, I_{p}^{i f}$ the corresponding ionization potential, and $\tilde{d}_{r}^{i f}$ the corresponding molecular-frame complex photorecombination matrix element. The transit time of the electron in the continuum amounts to $\tau \approx 0.8-1.7 \mathrm{fs}$ under our experimental conditions $[15,16]$. The quantities entering 

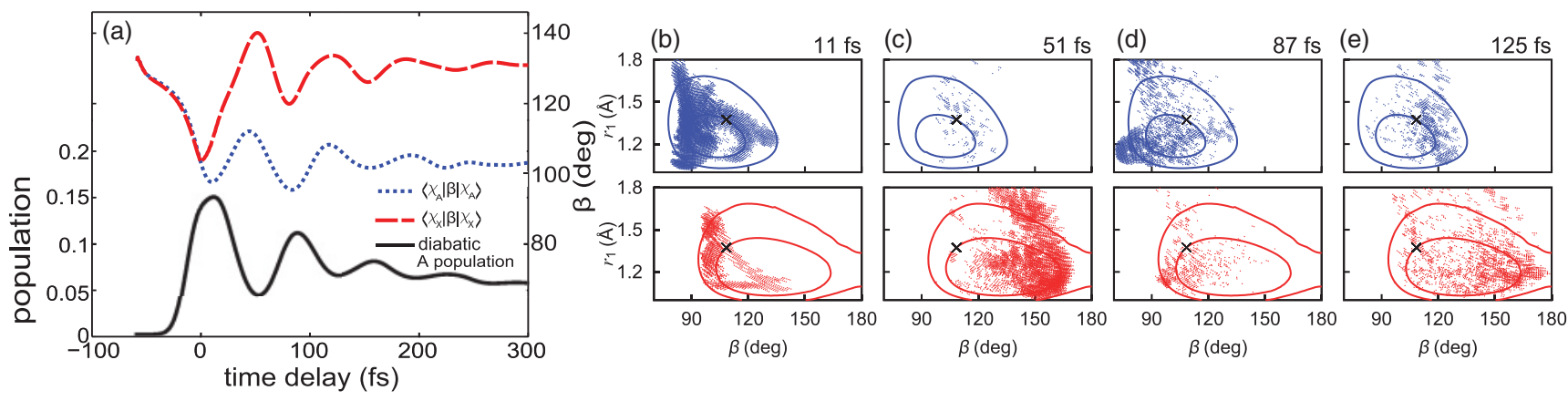

FIG. 3. (Color online) Results of a full-dimensional quantum dynamical wave-packet calculation on $\mathrm{NO}_{2}$. Panel (a) shows the temporal variation of the population in the diabatic $\tilde{A}^{2} B_{2}$ state and the expectation values for the bending angle for the two diabatic wave packets. Panels (b)-(e) show projections of the amplitudes of the diabatic wave packets onto the $\left(\beta, r_{1}\right)$ plane at the indicated times, the upper row corresponding to the $\tilde{A}^{2} B_{2}$ state and the lower one to the $\tilde{X}^{2} A_{1}$ state. Potential surface contours of the electronic states are $3.00 \mathrm{eV}$ (outer) and $1.77 \mathrm{eV}$ (inner), and the conical intersection is indicated by a cross for $r_{2}=1.37 \AA$.

Eq. (5) were obtained from quantum chemical ab initio calculations as described in Ref. [10]. Briefly, the quantum chemical calculations were performed at the $\operatorname{CASSCF}(17,12) / /$ augcc-pVTZ level of theory for three characteristic geometries of the $X$ and $A$ state, corresponding to bending angles of $85^{\circ}, 102^{\circ}$, and $134^{\circ}$ and relaxed bond-length coordinates. The strong-field ionization rates $I^{i f}$ have been calculated by solving the single-electron time-dependent Schrödinger equation in the ionic Hartree potential of the appropriate cation state [17] and the photorecombination matrix elements $\tilde{d}_{r}^{i f}$ have been calculated in the eikonal approximation using the Hartree potentials and Dyson orbitals [4,18]. The coordinate dependence of the vertical ionization potentials has been taken from Ref. [13].

Since the transition dipole moment for the $\tilde{A}^{2} B_{2} \leftarrow \tilde{X}^{2} A_{1}$ transition lies parallel to the $\mathrm{O}-\mathrm{O}$ axis ( $y$ axis in Fig. 1), only the $a_{1}$ orbital is ionized when pump and probe pulses are orthogonally polarized, whereas ionization from the $b_{2}$ orbital is strongly suppressed for symmetry reasons [10]. Consequently, molecules in the $X$ state ionize to the ${ }^{1} A_{1}$ state of the cation (channel XS in Fig. 1) whereas molecules in the $A$ state ionize to the ${ }^{3} B_{2}$ state of the cation (channel AT in Fig. 1). In the case of parallel polarizations, only the $b_{2}$ orbital ionizes significantly. Thus, molecules in the $X$ state are ionized to the ${ }^{3} B_{2}$ state (channel XT), whereas molecules in the $A$ state are ionized to the ${ }^{1} A_{1}$ state (channel AS), which is only possible in the region $85^{\circ}<\beta<105^{\circ}$ where the configuration $(\ldots)\left(a_{1}\right)^{2}\left(b_{2}\right)^{0}$ dominates.

To interpret the experimental results, we now study the mechanism causing the oscillations and their polarization dependence. Equation (4) shows that the calculated oscillations may have contributions from both the electronic populations dynamics $r_{i}(t)$ and from modulations of the high-harmonic emission dipole $\tilde{d}_{i}(t)$. To distinguish these two contributions, we model the dynamics using two limiting cases. In the first case, since bending dominates the early motion of the wave packet, we represent $\chi_{i}(\mathbf{R}, t)$ in Eq. (5) with Gaussian functions of FWHM $30^{\circ}$ in the bond-angle coordinate with their center moving as shown in Fig. 3(a). In the second case, we replace the coordinate- and hence time-dependent properties of high-harmonic emission with their average over the same Gaussian functions as above but localized at the minima of the respective diabatic states. The simulations of the first type are shown in Figs. 4(a) and 4(b) for the case of perpendicular and parallel polarizations, respectively, and the simulations of the second type are shown in panels 4(c) and 4(d). All simulations in Figs. 4(a)-4(d) predict counterphased oscillations in the diffracted and nondiffracted high-harmonic yields, with much deeper modulations in perpendicular than
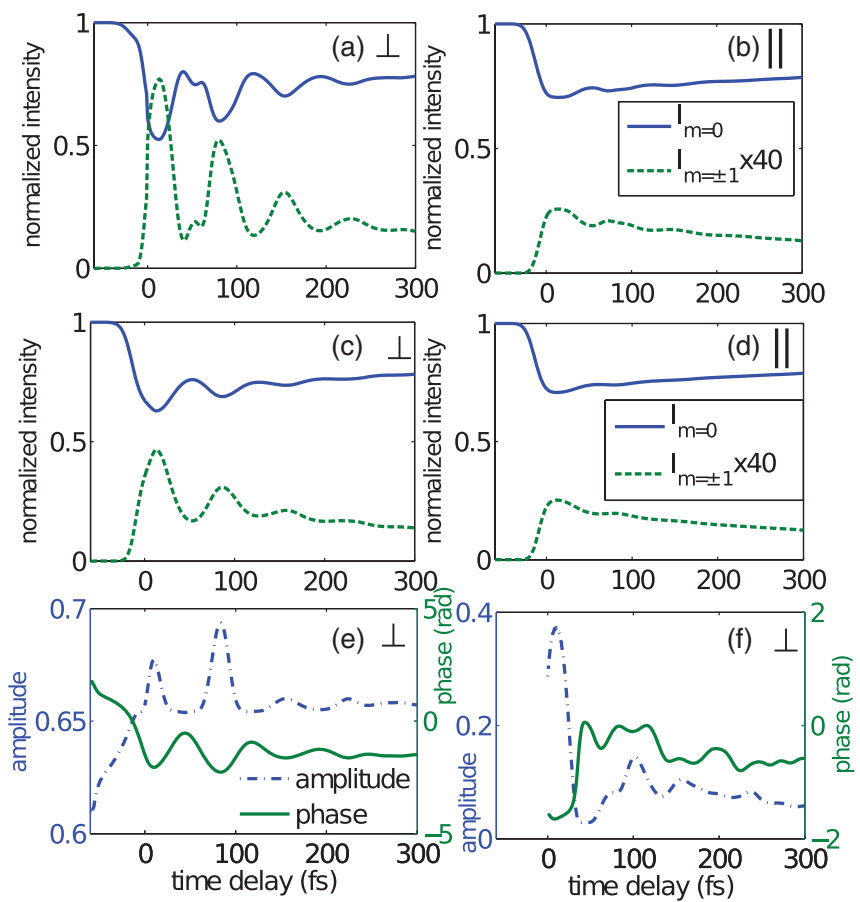

FIG. 4. (Color online) Results of the multichannel calculation of high-harmonic signals based on the wave-packet calculations for perpendicular and parallel polarizations of pump and probe pulses. In panels (a) and (b), the center of the wave packet has been allowed to move according to the wave-packet calculations and the coordinatespace integrals have been restricted to the bond angle. In panels (c) and (d) the motion of the wave packet has been neglected. Panel (e) shows the amplitude and phase of the propagation phase term $\left\langle\chi_{\mathrm{A}}\left|\mathrm{e}^{-i\left(I_{p}^{\mathrm{AT}}-I_{p}^{\mathrm{g}}\right) \tau}\right| \chi_{\mathrm{A}}\right\rangle$ averaged over the bending angle only. Panel (f) shows the same quantities for a three-dimensional coordinate-space average. 
in parallel polarizations, as observed in the experiment. The calculation in panel 4(a) significantly overestimates the modulation depth, but the calculation in panel $4(\mathrm{c})$ is in qualitative agreement with the experiment.

In previous work, we have shown that the variation of the vertical $I_{p}$ modulates the phase of $\tilde{d}_{i}(t)$, which highharmonic emission is highly sensitive to [5,6]. Consequently, we first study this effect in the present case. In perpendicular polarizations the AT channel has a phase shift $\Delta I_{p} \tau$ relative to unexcited molecules that varies almost linearly from +3 to -2 radians (for a typical value of $\tau=1.2 \mathrm{fs}$ ) over the range $85^{\circ}<\beta<134^{\circ}$ and thus deeply modulates the diffracted high-harmonic yield shown in Fig. 4(a). However, the significant spreading of the wave packet along the bond stretching coordinates and the associated distribution of ionization potentials has to be taken into account. We therefore compare the propagation phase term $\left\langle\chi_{\mathrm{A}}\left|\mathrm{e}^{-i\left(I_{p}^{\mathrm{AT}}-I_{p}^{\mathrm{g}}\right) \tau}\right| \chi_{\mathrm{A}}\right\rangle$ from the one-dimensional average over the bending coordinate with that from an average over the full three-dimensional coordinate space in Figs. 4(e) and 4(f). The strong phase modulations predicted by the one-dimensional average in panel 4(e), which lead to the deep modulations in panel 4(a), are averaged out in the three-dimensional calculation [panel 4(f)] as a consequence of the rapid spreading of the wave packet. The same is true for the amplitudes and phases of $\tilde{d}_{i}(t)$ for both electronic states and both polarization schemes.

Figures 4(c) and 4(d), which correspond to neglecting the wave-packet motion while retaining the electronic population dynamics, are in much closer agreement with the experimental observations for both polarization cases. Hence, we conclude that the observed and calculated oscillations reflect the temporal variation of the electronic population in the $A$ state. The difference in vertical $I_{p}$ values is thus only relevant in providing a time- and coordinate-averaged phase shift of the $A$-state emission relative to the unexcited molecules, but the observed oscillations reflect the diabatic population dynamics, or equivalently, the temporal variations of the electronic character of the excited molecules. The same argument holds for the case of parallel polarizations, where the temporal variation of the signal is also dominated by the electronic population dynamics.

In conclusion, we have shown that time-resolved highharmonic spectroscopy is a powerful method for probing the electronic dynamics associated with nonadiabatic dynamics in molecules. The sensitivity of the method to variation of the vertical ionization potentials will be most relevant when the wave packet remains localized along the coordinate(s) that strongly modulate(s) the $I_{p}$, like in diatomic molecules $[5,6]$ or in polyatomic molecules with a single soft coordinate [3]. On longer time scales, and more generally in larger polyatomic molecules, the sensitivity of the method to electronic population dynamics is expected to dominate. This sensitivity may become a powerful asset in studying electronic dynamics in photochemical switches or during pericyclic reactions. We note that in the case of $\mathrm{NO}_{2}$, the sensitivity to the population dynamics comes almost exclusively from the time- and coordinate-averaged difference in $I_{p}$ between the AT and XS channels. This is the case because the two channels correspond to ionization from the same orbital. However, the ground and excited states will in general ionize from different orbitals, in which case the method will be sensitive to differences in the phase and amplitude of recombination matrix elements, as well as the ionization rates, giving direct access to the evolving electronic structure of the transient molecule.

This work was supported by the Swiss National Science Foundation under Grant No. PP00P2_128274 and ETH Zürich under Grant No. ETH-33 10-3.
[1] J. Itatani, J. Levesque, D. Zeidler, Hiromichi Niikura, H. Pépin, J. C. Kieffer, P. B. Corkum, and D. M. Villeneuve, Nature (London) 432, 867 (2004).

[2] S. Baker, J. S. Robinson, C. A. Haworth, H. Teng, R. A. Smith, C. C. Chirilă, M. Lein, J. W. G. Tisch, and J. P. Marangos, Science 312, 424 (2006).

[3] W. Li, X. Zhou, R. Lock, S. Patchkovskii, A. Stolow, H. C. Kapteyn, and M. M. Murnane, Science 322, 1207 (2008).

[4] O. Smirnova, Y. Mairesse, S. Patchkovskii, N. Dudovich, D. Villeneuve, P. Corkum, and M. Yu. Ivanov, Nature (London) 460, 972 (2009).

[5] H. J. Wörner, J. B. Bertrand, P. B. Corkum, and D. M. Villeneuve, Phys. Rev. Lett. 105, 103002 (2010).

[6] H. J. Wörner, J. B. Bertrand, D. V. Kartashov, P. B. Corkum, and D. M. Villeneuve, Nature (London) 466, 604 (2010).

[7] V.-H. Le, A.-T. Le, R.-H. Xie, and C. D. Lin, Phys. Rev. A 76, 013414 (2007).

[8] A.-T. Le, R. R. Lucchese, S. Tonzani, T. Morishita, and C. D. Lin, Phys. Rev. A 80, 013401 (2009).
[9] M. V. Frolov, N. L. Manakov, T. S. Sarantseva, M. Y. Emelin, M. Y. Ryabikin, and A. F. Starace, Phys. Rev. Lett. 102, 243901 (2009).

[10] H. J. Wörner, J. B. Bertrand, B. Fabre, J. Higuet, H. Ruf, A. Dubrouil, S. Patchkovskii, M. Spanner, Y. Mairesse, V. Blanchet et al., Science 334, 208 (2011).

[11] I. Wilkinson and B. J. Whitaker, Annu. Rep. Prog. Chem., Sect. C: Phys. Chem. 106, 274 (2010).

[12] J. B. Bertrand, H. J. Worner, H. C. Bandulet, E. Bisson, M. Spanner, J. C. Kieffer, D. M. Villeneuve, and P. B. Corkum, Phys. Rev. Lett. 106, 023001 (2011).

[13] Y. Arasaki and K. Takatsuka, Chem. Phys. 338, 175 (2007).

[14] Y. Arasaki, K. Takatsuka, K. Wang, and V. McKoy, J. Chem. Phys. 132, 124307 (2010).

[15] M. Lewenstein, P. Balcou, M. Y. Ivanov, A. L'Huillier, and P. B. Corkum, Phys. Rev. A 49, 2117 (1994).

[16] T. Kanai, E. J. Takahashi, Y. Nabekawa, and K. Midorikawa, Phys. Rev. Lett. 98, 153904 (2007).

[17] M. Spanner and S. Patchkovskii, Phys. Rev. A 80, 063411 (2009).

[18] S. Patchkovskii, Z. Zhao, T. Brabec, and D. M. Villeneuve, J. Chem. Phys. 126, 114306 (2007). 